\title{
The Thermal and Mechanical Properties and the Ionic Conductivity of Cationic Polyurethane Elastomers with Pendant Trimethylammonium Group
}

\author{
Kyoko HiRAOKA ${ }^{\dagger}$, Masafumi TAKESUE* ${ }^{*}$, and Tetsuo YoKOYAMA** \\ Faculty of Environmental Studies, Nagasaki University, 1-14 Bunkyo-machi, Nagasaki 852-8521, Japan \\ *Bando Chemical Industries, 3-1-6 Ashihara-dori, Hyogoku, Kobe 652-0882, Japan \\ **Tamaki Women's College, 1-33 Kazagashira, Nagasaki 850-0803, Japan
}

(Received March 14, 2003; Accepted October 24, 2003)

\begin{abstract}
A series of cationic polyurethane (PU) elastomers with pendant ammonium group, different polyether chains, and different content of ionic groups were prepared by nonsolvent technique. NCO-terminated prepolymers were prepared from 2,4-tolylene diisocyanate and polyols including poly(oxytetramethylene)glycol (PTMG) and poly(oxypropylene) glycol (PPG). Cationic PU elastomers were prepared by the reaction of prepolymer with the mixture of chain extenders [1,1,1-trimethylolpropane, 2-ethyl-2-methyl-1,3-propanediol, and cationic diol, 3-trimethylammonium(1,2-propanediol)iodide]. The properties of cationic PU elastomers were studied regarding the effect of polyol type, ion content, and ion site by means of differential scanning calorimetry, dynamic mechanical thermal analysis, tensile testing, and complex impedance measurement. As the ion content increased, the demixing of hard segment from PTMG soft segment proceeded. PTMG-rich soft micro-phase became purer but its quantity decreased. The trend was reduced for PPG-based PU elastomers. While PU elastomers with ammonium group in pendant have micro-multiphase structure, PU elastomers with that in backbone have approximately homogeneous structure. The ionic conductivity was about $10^{-10} \mathrm{~S} \mathrm{~cm}^{-1}$ at room temperature and $10^{-7} \mathrm{~S} \mathrm{~cm}^{-1}$ at $100{ }^{\circ} \mathrm{C}$. The effects of micro-phase separation on the conductivity of cationic PU elastomers were discussed. Temperature dependence of conductivity obeyed Vogel-TammanFulcher equation.

KEY WORDS Cationic Polyurethane / Pendant Ammonium Group / Heterogeneity / Thermal Property / Mechanical Property / Ionic Conductivity /
\end{abstract}

Polyurethanes (PUs) are very versatile industrial materials which have been developed during these seventy years and have been applied in various fields as foams, coatings, adhesives, elastomers, fibers, synthetic leathers, etc. Even on elastomers, PUs have been applied in many fields by putting its superior mechanical properties, abrasion resistance, and oil resistance to practical use. Elastomers are insulating material in general and PU elastomers also. However, as the electronic devices advance in recent years, elastomers used for roller and cleaning blade inside of electronic camera have been needed to have the conductivity of semiconductor level $\left(10^{-7}-10^{-10} \mathrm{~S} \mathrm{~cm}^{-1}\right)$. In such applications, PU elastomers have been used for their good abrasion resistance and low compression set. It is considerable that PU elastomers will be needed to have conductivity of much higher level in future.

One method to improve conductivity of PU elastomers is to introduce ionic conductivity. In general, polyether-based PU elastomers can be complexed with electrolytes such as $\mathrm{LiClO}_{4}$ to give ionic conductivity. There have been various investigations of PUelectrolyte complexes. ${ }^{1,2}$ However, the use of high concentration of electrolytes leads to the loss of elasticity. The use of solvent is usually essential to complexing PU elastomers with electrolytes, which is disadvantage for the industrial production. Other semiconductor type of PU elastomers is ionic PU elastomers.

PU ionomers have been the important field of ionic PU elastomers. In general, ionomers are thermoplastic materials containing up to $\sim 10 \%$ of ionic comonomers and are thus insoluble in water in most cases. PU ionomers are classified into (a) PU cationomers ${ }^{3-7}$ and (b) PU anionomers ${ }^{8,9}$ depending upon whether positive or negative ion is contained in main chain or pendant chain, and (c) PU zwitter ionomers ${ }^{10}$ containing both the positive and negative ions.

For the syntheses of PU cationomers, it is the popular route to use the chain extenders of short-chain diols containing tertiary amine group, and quarternize the formed PU with acids or alkyl halides. ${ }^{3,11}$ However, the use of solvent is inevitable in this route. In recent studies ${ }^{6,8,12}$ synthetic route that uses prequarternized diol is adopted. The advantages of prequarternization before the formation of PU elastomers are the realization of synthesis in nonsolvent system

${ }^{\dagger}$ To whom correspondence should be addressed (E-mail: hiraoka@ net.nagasaki-u.ac.jp). 
Table I. Materials

\begin{tabular}{ccl}
\hline Abbreviation & Description & \multicolumn{1}{c}{ Supplier } \\
\hline TDI & 2,4-Tolylene diisocyanate & Nippon Polyurethane \\
& & Industry Co., Ltd. \\
PTMG & Poly(oxytetramethylene) glycol & Hodogaya Chemical \\
& $\bar{M}_{\mathrm{n}}=980$ & Co., Ltd. \\
PPG & Poly $($ oxypropylene) glycol & Sanyo Kasei Industry \\
& $\bar{M}_{\mathrm{n}}=880$ & Co., Ltd. \\
TMP & 1,1,1-Trimethylol propane & Tokyo Kasei \\
& & Kogyo Co., Ltd. \\
EMP & 2-Ethyl-2-methyl-1,3-propanediol & Tokyo Kasei \\
& & Kogyo Co., Ltd. \\
BD & 1,4-Butanediol & Wako Pure Chemical \\
& & Industies, Ltd. \\
DMP & Tokyo Kasei \\
& 3-Dimethylamino-1,2-propanediol & Kogyo Co., Ltd. \\
MEA & & Wako Pure Chemical \\
& $N$-Methyldiethanolamine & Industies, Ltd. \\
& & Wako Pure Chemical \\
& Methyl iodide & Industies, Ltd. \\
\hline
\end{tabular}

and the easy control of content of ionic group in PU elastomers. Several researchers have investigated structure-property relationships in such systems, where the quaternary ammonium group is in the PU backbone.

Short-chain diols with pendant pyridinium halides were used by Vilenskii et al. ${ }^{13}$ to synthesize PU cationomers directly. The effects of the neutralizing anion on polymer morphology and thermal properties were investigated. Varma and co-workers ${ }^{14}$ have prepared PU cationomers by using 3-trimethylammonium(1,2-propanediol)iodide as the chain extender, but properties of the obtained PU cationomers were not reported. Goddard and Cooper ${ }^{15-17}$ have synthesized PU cationomers with pendant trialkylammonium groups by using ionic chain extenders. They have investigated the morphology and properties of their PU cationomers as a function of ion content, the length of alkyl group, and neutralizing anion.

As described above, there have been various investigations of PU ionomers, which are thermoplastic polymers. However, very little work is currently available in the published literature on the thermosetting ionic PU elastomers. The research of the thermosetting ionic PU elastomers is needed in PU industry with anticipation of their improved properties. On the other hand, we have reported the synthesis and properties of thermosetting cationic PU elastomers that were prepared by using ionic chain extender with quaternary ammonium group in backbone. ${ }^{18,19}$

On the background described above, this study intends (1) to use cationic short-chain diol with pendant quaternary ammonium group as chain extender, (2) to synthesize cationic PU elastomers containing different polyethers and different content of ionic group by nonsolvent technique, and (3) to examine the thermal and mechanical properties, and the ionic conductivity of the obtained cationic PU elastomers, and to compare with the corresponding cationic PU elastomers with quaternary ammonium group in backbone.

\section{EXPERIMENTAL}

\section{Materials}

Materials used in this study are described in Table I with their abbreviations. Polyols were dried and degassed at $65^{\circ} \mathrm{C}$ under vacuum for $3 \mathrm{~h}$ before use. Chain extenders were dried likewise before use. Other materials were used as received.

\section{Preparation of Cationic Diols Containing Quaternary Ammonium Group}

Methyl iodide was added slowly with stirring to 3dimethylamino-1,2- propanediol (DMP) or $N$-methyldiethanolamine (MEA) at room temperature under nitrogen atmosphere to yield 3-trimethylammonium(1,2-propanediol) iodide (TMPI) or $N$-dimethyldiethanolammonium iodide (MEAI). The detail of the preparation was described in the previous paper. ${ }^{19}$ The formation of TMPI and MEAl were confirmed by the results of elementary analysis and NMR spectra.

\section{Preparation of Cationic Polyurethane Elastomers}

Cationic PU elastomers with pendant ammonium group were synthesized by a two-step addition reaction outlined in Figure 1. The NCO-terminated prepolymers were prepared by the reaction of TDI with polyols at a molar ratio of $\mathrm{NCO} / \mathrm{OH}=2 / 1$ under dry nitrogen atmosphere for 15-30 min. Polyols used were PTMG and PPG. The reaction was carried out at 65- 


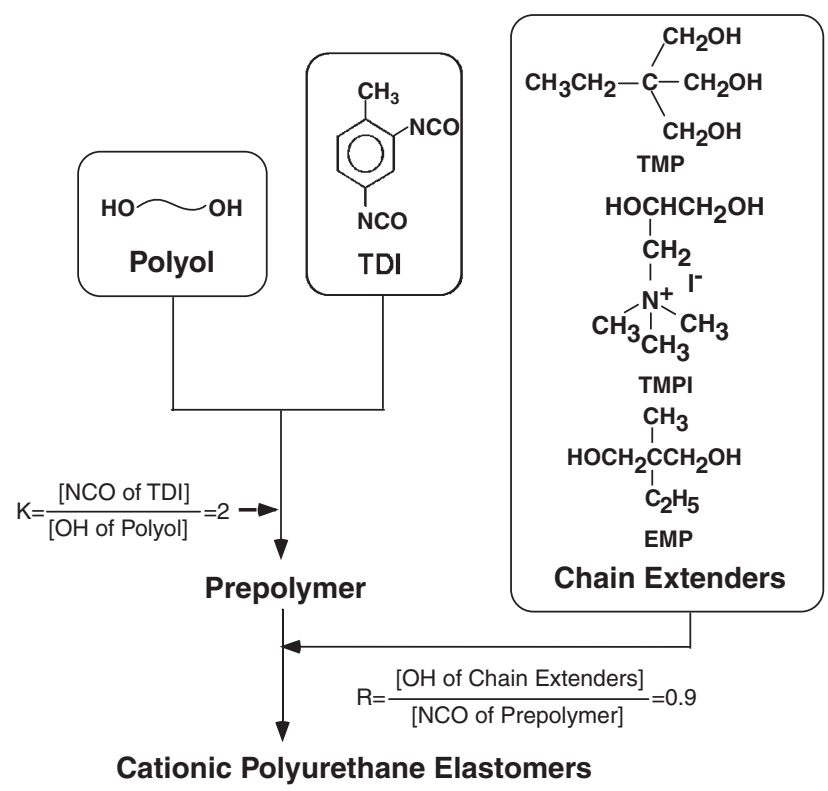

Figure 1. Two-step synthesis of cationic polyurethane elastomers.

$75^{\circ} \mathrm{C}$ without any catalyst, and terminated when the resulting isocyanate content of prepolymer determined by the amine equivalent method reached expected value. After the prepolymer was degassed under vacuum to remove any possible air bubbles, the mixture of chain extenders (TMP, EMP, and TMPI) was added to the prepolymer at a molar ratio $\mathrm{OH} /$ $\mathrm{NCO}=0.9$. The reaction was carried out at 80 $100{ }^{\circ} \mathrm{C}$ for $1-10 \mathrm{~min}$ with high speed stirring, then the reaction mixture was cast in a mold kept at $100{ }^{\circ} \mathrm{C}$. After standing in an oven at $100^{\circ} \mathrm{C}$ for $1-$ $3 \mathrm{~h}$, the cationic PU elastomer was demolded, and then post-cured in a oven at $100^{\circ} \mathrm{C}$ for $24 \mathrm{~h}$. The composi- tion of mixed chain extenders was as follows, whose $30 \mathrm{~mol} \%[\mathrm{OH}]$ was of TMP, and other $[\mathrm{OH}]$ was of EMP and TMPI. The degree of ionization of the samples was controlled by regulating the fraction of EMP and TMPI. Cationic PU elastomers with ammonium group in backbone were prepared by using BD and MEAI as substitutes for EMP and TMPI, respectively. The composition ratios of the chain extenders are shown in Table II.

The samples were designated in the following manner: 10T-PN-0.6. The first alphabet of the mnemonic code refers to the polyol type ("T" for PTMG and "P" for PPG), the second alphabet refers to ionic chain extender ("PN" for TMPI, "BN" for MEAI: $\mathrm{PN}$ means pendant $\mathrm{N}^{+}$and $\mathrm{BN}$ backbone $\mathrm{N}^{+}$); the first figures, 10, indicates the molecular weight 1000 of the polyols, and the last figures is the weight percentage of quaternary ammonium calculated from theoretical values.

\section{Characterization}

Equilibrium water sorption in 20 and $90 \%$ relative humidity at $25^{\circ} \mathrm{C}$ was measured by weighing at an appropriate time interval until constant weight was reached. The percentage of water sorption was calculated from the weight difference between the wet and dry samples.

Differential scanning calorimetry (DSC) was carried out with a Seiko DSC 210 at the heating rate of $10^{\circ} \mathrm{C} \mathrm{m^{-1 }}$.

Rheometrics RSA II (Rheometrics for East) was used to obtain dynamic mechanical data at temperatures ranging from -150 to $200^{\circ} \mathrm{C}$ at $0.1 \%$ of strain, the frequency of $10 \mathrm{~Hz}\left(62.8 \mathrm{rad} \mathrm{s}^{-1}\right)$, and the heating

Table II. Compositions and various properties of cationic polyurethane elastomers

\begin{tabular}{|c|c|c|c|c|c|c|c|c|c|c|}
\hline \multirow[t]{2}{*}{ Sample code } & \multicolumn{5}{|c|}{$\begin{array}{l}\text { Composition } \\
(\mathrm{OH} \text { mol \%) }\end{array}$} & \multirow{2}{*}{$\begin{array}{l}{\left[\mathrm{N}^{+}\right]} \\
(\mathrm{wt} \%)\end{array}$} & \multirow{2}{*}{$\begin{array}{l}\text { [ether group] } \\
\left(\times 10^{-4} \mathrm{~mol} / \mathrm{g}\right)\end{array}$} & \multirow[t]{2}{*}{ Appearance } & \multicolumn{2}{|c|}{$\begin{array}{l}\text { Water sorption } \\
\text { (wt } \%)\end{array}$} \\
\hline & TMP & EMP & TMPI & $\mathrm{BD}$ & MEAI & & & & $20 \mathrm{RH} \%$ & $90 \mathrm{RH} \%$ \\
\hline 10T-PN-0 & 30 & 70 & 0 & & & 0 & 87 & Colorless, transparent & 1.3 & 3.2 \\
\hline 10T-PN-0.2 & 30 & 45 & 25 & & & 0.20 & 85 & Dark yellow, opaque & 1.4 & 3.5 \\
\hline 10T-PN-0.4 & 30 & 25 & 45 & & & 0.37 & 84 & Dark yellow, opaque & 1.7 & 3.9 \\
\hline 10T-PN-0.6 & 30 & 0 & 70 & & & 0.58 & 82 & Dark yellow, opaque & 1.7 & 4.6 \\
\hline 10T-BN-0 & 30 & & & 70 & 0 & 0 & 88 & Colorless, transparent & 1.0 & 2.7 \\
\hline 10T-BN-0.2 & 30 & & & 45 & 25 & 0.21 & 85 & White, translucent & 1.2 & 3.3 \\
\hline 10T-BN-0.4 & 30 & & & 25 & 45 & 0.39 & 83 & White, opaque & 1.2 & 4.0 \\
\hline 10T-BN-0.6 & 30 & & & 0 & 70 & 0.58 & 81 & White, opaque & 1.3 & 4.7 \\
\hline 10P-PN-0 & 30 & 70 & 0 & & & 0 & 103 & Colorless, transparent & 1.0 & 3.7 \\
\hline 10P-PN-0.2 & 30 & 45 & 25 & & & 0.23 & 100 & Yellow, translucent & 1.0 & 4.9 \\
\hline 10P-PN-0.4 & 30 & 25 & 45 & & & 0.42 & 98 & Yellow, opaque & 1.3 & 5.7 \\
\hline 10P-PN-0.6 & 30 & 0 & 70 & & & 0.63 & 96 & Yellow, opaque & 1.5 & 6.8 \\
\hline 10P-BN-0 & 30 & & & 70 & 0 & 0 & 104 & Colorless, transparent & 0.9 & 4.1 \\
\hline 10P-BN-0.2 & 30 & & & 45 & 25 & 0.23 & 101 & Light yellow, translucent & 1.2 & 5.2 \\
\hline 10P-BN-0.4 & 30 & & & 25 & 45 & 0.42 & 99 & Yellow, translucent & 1.1 & 5.4 \\
\hline 10P-BN-0.6 & 30 & & & 0 & 70 & 0.62 & 96 & Yellow, translucent & 1.3 & 6.0 \\
\hline
\end{tabular}


rate of $2{ }^{\circ} \mathrm{C} \mathrm{min}^{-1}$. The size of test piece was $40 \mathrm{~mm}$ long, $5 \mathrm{~mm}$ wide, and $1 \mathrm{~mm}$ thick.

Hardness was measured by means of ASKER-A Durometer (Koubunshi Keiki Co.) according to JISK6301.

Tensile strength and elongation at break were measured by means of a Shimadzu Universal Testing Machine, Model AGS-100 at a crosshead speed of $500 \mathrm{~mm} \mathrm{~min}^{-1}$ (JIS-K6301). The standard dumbbellshaped die III was used to cut specimen from the test sheets.

Ionic conductivity was determined by complex impedance measurement. The impedance measurements with alternating current were carried out with 5020 frequency response analyzer (NF Electric instruments) — a potentiostat/galvanostat 2000 (TOHO technical research) system in the frequency range from $10 \mathrm{mHz}$ to $20 \mathrm{kHz}$. The test sheets $(1 \mathrm{~mm}$ thick) were cut to $25 \times 25 \mathrm{~mm}$ size and then Au was deposited at both sides to form two Au electrodes (the diameter is $c a .11 \mathrm{~mm}$ ). The test specimen was kept in a temperature-controlled glass vessel, which was flushed with nitrogen gas. The temperature dependence of the conductivity was measured in the range from room temperature to around $150^{\circ} \mathrm{C}$. The ionic conductivity of the samples was calculated by eq. 1

$$
\sigma=\frac{d}{R_{\mathrm{B}} S}
$$

where $d(\mathrm{~cm})$ is the thickness of the samples, $S\left(\mathrm{~cm}^{2}\right)$ is the area of the Au electrode, and $R_{\mathrm{B}}(\Omega)$ is the bulk resistance of the samples. The test specimens were dried at $50^{\circ} \mathrm{C}$ in a vacuum oven over $2 \mathrm{~h}$ before all measurements.

\section{RESULTS}

\section{Appearance and State of Samples}

The appearance of the samples was summarized in Table II. All the samples were elastomers at room temperature. The transparency of the samples decreased with increasing degree of ionization. The degree of transparency varied with polyols and chain extenders.

The unionized samples are colorless and transparent, suggesting little or no aggregation of hard segments. With increasing ion content, PN series lost transparency at lower ion content than $\mathrm{BN}$ series.

\section{Water Sorption}

Table II also shows the percentage of water sorption of the samples. The water sorption increased with increasing ionization for all the samples measured. This is clear evidence that ionic groups were incorporated systematically into the cationic PU elastomers. Though all the samples show similarly low values of

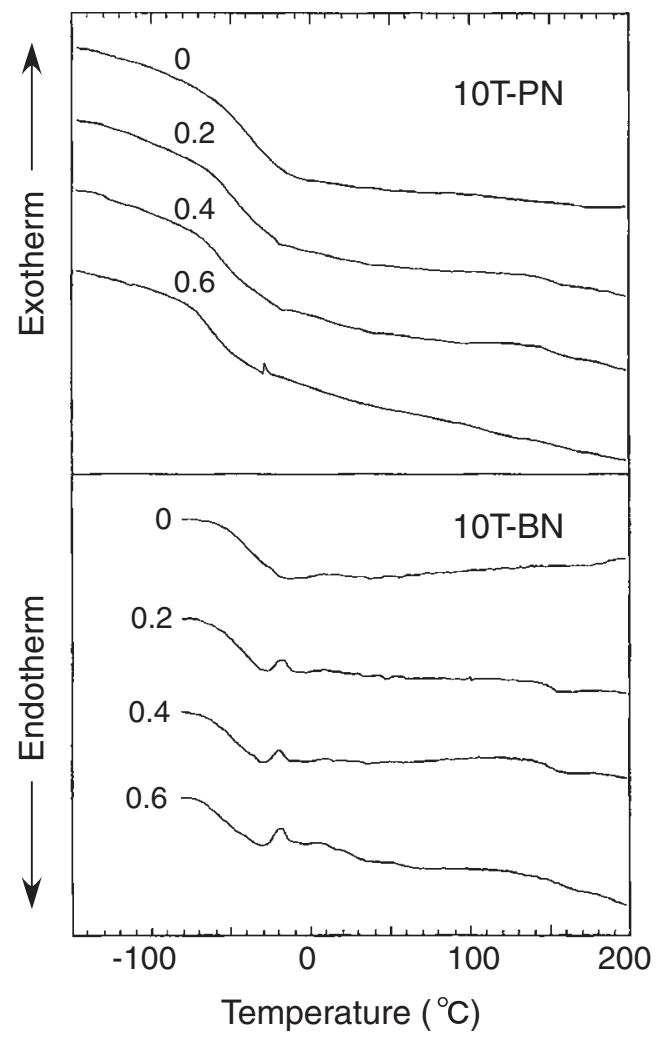

Figure 2. DSC traces of $10 \mathrm{~T}-\mathrm{PN}$ and $10 \mathrm{~T}-\mathrm{BN}$ series with the ion contents indicated.

water sorption when relative humidity is low, 10P series show larger values than those of $10 \mathrm{~T}$ series at the corresponding ion content when relative humidity is high. The order, PPG $>$ PTMG, agrees with the ether concentration of the samples. The position of ammonium groups, whether in pendant or in backbone, had no effect on water sorption.

\section{Differential Scanning Calorimetry}

DSC thermograms for 10T series are shown in Figure 2. The thermal transition data for all the samples are given in Table III.

It was observed that each sample exhibited single glass transition based on soft segment transition. 10T-BN series and 10T-PN-0.6 showed, in addition to $T_{\mathrm{g}}$, a small exothermic peak near -20 or $-30^{\circ} \mathrm{C}$ respectively, indicating recrystallization of the PTMG segments. The peak temperature of recrystallization of $\mathrm{BN}$ series is slightly higher than that of PN-0.6. This may be the result of enhanced mixing of PTMG with ionic segment, which will delay the beginning of micro-Brownian motion of chain. The peak area is apparently large for $\mathrm{BN}$ series. That is, 10T-BN series recrystallize more easily than 10T-PN series after glass transition. This is probably because the constraint of chain by aggregation of hard segments in $\mathrm{BN}$ series is weaker than in PN series. 10P series (not shown in Figure 2) based on noncrystalline PPG naturally showed no recrystallization peak. 
Table III. Thermal transition data

\begin{tabular}{|c|c|c|c|c|}
\hline \multirow[b]{2}{*}{ Sample } & \multicolumn{3}{|c|}{ DSC Results } & \multirow{2}{*}{$\begin{array}{c}\text { DMTA Results } \\
T_{\alpha} \\
\left({ }^{\circ} \mathrm{C}\right)\end{array}$} \\
\hline & $\begin{array}{c}T_{\mathrm{g}} \\
\left({ }^{\circ} \mathrm{C}\right)\end{array}$ & $\begin{array}{l}\Delta T_{\mathrm{g}} \\
\left({ }^{\circ} \mathrm{C}\right)\end{array}$ & $\begin{array}{c}\Delta C p \\
(\mathrm{~J} / \mathrm{K} \cdot \mathrm{g})\end{array}$ & \\
\hline 10T-PN-0 & -41.9 & 49.9 & 0.75 & -27 \\
\hline 10T-PN-0.2 & -45.8 & 40.9 & 0.53 & -39 \\
\hline 10T-PN-0.4 & -51.3 & 37.6 & 0.47 & -43 \\
\hline 10T-PN-0.6 & -60.7 & 31.7 & 0.44 & -45 \\
\hline 10T-BN-0 & -41.1 & 35.8 & 0.60 & -31 \\
\hline 10T-BN-0.2 & -48.2 & 31.6 & 0.49 & -39 \\
\hline $10 \mathrm{~T}-\mathrm{BN}-0.4$ & -51.3 & 32.2 & 0.47 & -43 \\
\hline 10T-BN-0.6 & -55.2 & 34.4 & 0.44 & -47 \\
\hline 10P-PN-0 & -8.2 & 18.6 & 0.63 & -3 \\
\hline 10P-PN-0.2 & -11.3 & 22.5 & 0.66 & -7 \\
\hline 10P-PN-0.4 & -11.4 & 34.4 & 0.61 & -7 \\
\hline 10P-PN-0.6 & -12.9 & 33.8 & 0.55 & -7 \\
\hline 10P-BN-0 & -12.9 & 18.1 & 0.69 & -7 \\
\hline 10P-BN-0.2 & -14.5 & 21.0 & 0.79 & -11 \\
\hline 10P-BN-0.4 & -15.3 & 25.7 & 0.74 & -11 \\
\hline 10P-BN-0.6 & -14.5 & 41.4 & 0.79 & -11 \\
\hline
\end{tabular}

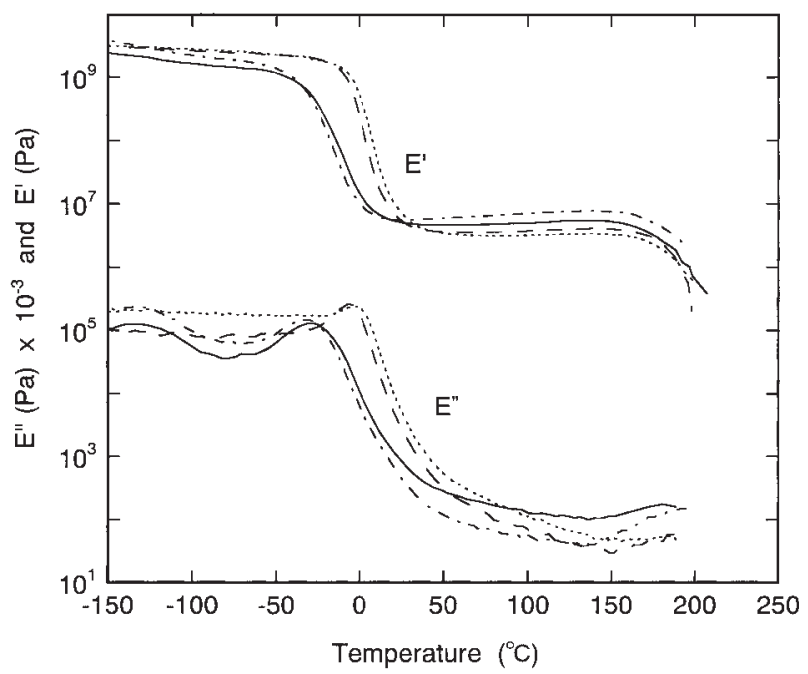

Figure 3. $E^{\prime}$ and $E^{\prime \prime}$ for unionized polyurethane elastomers: 10T-PN; ------ 10T-BN; ……... 10P-PN; ----10P-BN.

\section{Dynamic Mechanical Thermal Analysis}

The effects of ion content, pendant ammonium group, and polyether species on dynamic mechanical properties are illustrated in Figures 3 and 4. Recrystallization of PTMG segment which was detected in DSC measurement was not reflected in DMTA because its extent was small. $T_{\alpha}$, the peak temperatures of the loss modulus $\left(E^{\prime \prime}\right)$, which correspond to the $T_{\mathrm{g}}$ of the soft segment, is summarized in Table III.

As for the loss modulus $\left(E^{\prime \prime}\right)$, 10T series exhibited a small peak at $-135^{\circ} \mathrm{C}$. This peak is attributable to the crankshaft motion of methylene sequence in PTMG segments. This peak was not observed for 10P series.

As shown in Figure 4, ionization results in a broad-

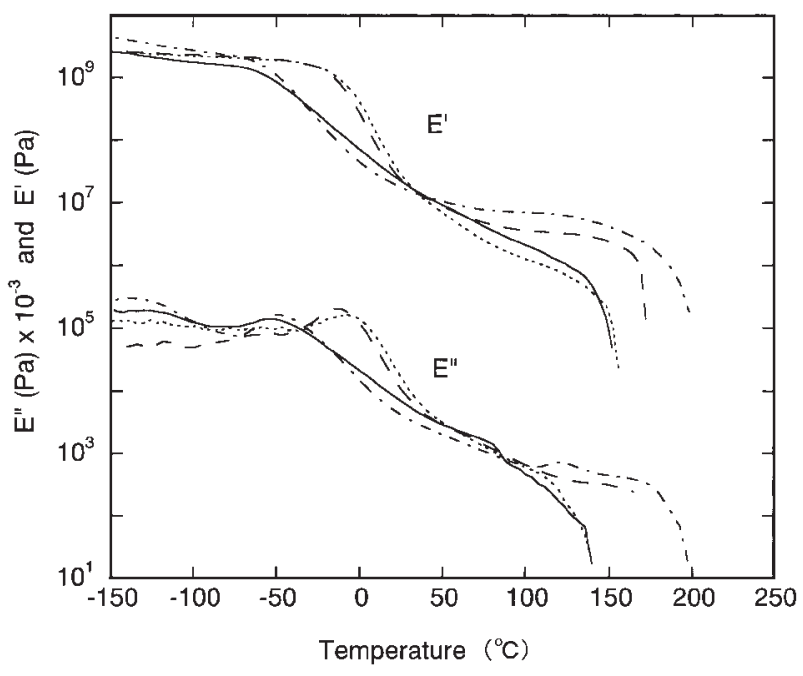

Figure 4. $E^{\prime}$ and $E^{\prime \prime}$ for cationic polyurethane elastomers with 0.6 ionization level: $-10 \mathrm{~T}-\mathrm{PN} ;-$ - - - - 10T-BN; ........... 10P-PN; ----- 10P-BN.

Table IV. Mechanical properties

\begin{tabular}{lccc}
\hline \multicolumn{1}{c}{ Sample } & $\begin{array}{c}\text { Hardness } \\
\text { (JIS-A) }\end{array}$ & $\begin{array}{c}\text { Tensile } \\
\text { Strength } \\
(\mathrm{Mpa})\end{array}$ & $\begin{array}{c}\text { Ultimate } \\
\text { Elongation } \\
(\%)\end{array}$ \\
\hline 10T-PN-0 & 59 & 20 & 370 \\
10T-PN-0.2 & 60 & 26 & 590 \\
10T-PN-0.4 & 65 & 27 & 590 \\
10T-PN-0.6 & 72 & 21 & 710 \\
\hline 10T-BN-0 & 66 & 8.7 & 390 \\
10T-BN-0.2 & 68 & 15 & 380 \\
10T-BN-0.4 & 74 & 34 & 410 \\
10T-BN-0.6 & 81 & 39 & 400 \\
\hline 10P-PN-0 & 56 & 11 & 470 \\
10P-PN-0.2 & 60 & 8.5 & 480 \\
10P-PN-0.4 & 63 & 8.0 & 610 \\
10P-PN-0.6 & 69 & 10 & 590 \\
\hline 10P-BN-0 & 55 & 4.9 & 320 \\
10P-BN-0.2 & 54 & 11 & 430 \\
10P-BN-0.4 & 63 & 31 & 500 \\
10P-BN-0.6 & 69 & 43 & 460 \\
\hline
\end{tabular}

er transition and narrower or diminished rubbery plateau, which indicates the samples have multiphase structure. The behavior of the samples of 0.2 and 0.4 series was intermediate between that of unionized polymers and 0.6 series.

\section{Mechanical Properties}

The hardness, the tensile strength, and the elongation at break of the samples are shown in Table IV.

The increase of hardness with increasing ionization is considered to be the results of pseudo-crosslinking effect due to coulomb interaction, and reinforcement effect due to the aggregation of ion-containing segment.

The tensile strengths of 10T series are generally 
Table V. Ionic conductivity of cationic poyurethane elastomers

\begin{tabular}{lcc}
\hline \multirow{2}{*}{ Sample code } & \multicolumn{2}{c}{ Conductivity $\left(\mathrm{S} \mathrm{cm}^{-1}\right)$} \\
\cline { 2 - 3 } & at $25^{\circ} \mathrm{C}$ & at $100^{\circ} \mathrm{C}$ \\
\hline 10T-PN-0.2 & $7.8 \times 10^{-11}$ & $4.6 \times 10^{-8}$ \\
10T-PN-0.4 & $1.2 \times 10^{-10}$ & $7.8 \times 10^{-8}$ \\
10T-PN-0.6 & $1.1 \times 10^{-10}$ & $1.1 \times 10^{-7}$ \\
\hline 10T-BN-0.2 & $4.8 \times 10^{-10}$ & $5.6 \times 10^{-8}$ \\
10T-BN-0.4 & $3.1 \times 10^{-10}$ & $1.6 \times 10^{-7}$ \\
10T-BN-0.6 & $1.7 \times 10^{-10}$ & $1.2 \times 10^{-7}$ \\
\hline 10P-PN-0.2 & $1.0 \times 10^{-11}$ & $6.9 \times 10^{-8}$ \\
10P-PN-0.4 & $4.7 \times 10^{-11}$ & $1.7 \times 10^{-7}$ \\
10P-PN-0.6 & $5.4 \times 10^{-11}$ & $1.8 \times 10^{-7}$ \\
\hline 10P-BN-0.2 & $5.5 \times 10^{-11}$ & $8.8 \times 10^{-8}$ \\
10P-BN-0.4 & $1.0 \times 10^{-10}$ & $1.7 \times 10^{-7}$ \\
10P-BN-0.6 & $1.5 \times 10^{-10}$ & $2.6 \times 10^{-7}$ \\
\hline
\end{tabular}

higher than those of 10P series. The order is reasonable, since the crystallization due to orientation of PTMG chain on stretching is easier than that of PPG chain. As for BN series, increasing the ionization results in increasing tensile strength. As for PN series, tensile strength remained at almost the same level with increasing ionization. This is because the measurement temperature of mechanical properties was room temperature, which is located in transition region.

PN series showed greater values of elongation at break in comparison with $\mathrm{BN}$ series.

\section{Ionic Conductivity}

The unionized samples have so great complex impedance that this exceeds the measuring limit. The Cole-Cole plot did not show full semicircle until temperature was elevated. From high temperature measurement, the ionic conductivity was presumed to be $10^{-14}-10^{-12} \mathrm{~S} \mathrm{~cm}^{-1}$.

Table $\mathrm{V}$ shows the ionic conductivity of the samples at 25 and $100^{\circ} \mathrm{C}$, and Figure 5 shows the temperature dependence of the ionic conductivity of 0.6 series. The other series showed similar dependence.

\section{DISCUSSION}

\section{Microstructure of Unionized Samples}

The appearances of unionized samples are colorless and transparent without regard to $10 \mathrm{~T}$ or $10 \mathrm{P}$ and to $\mathrm{PN}$ or BN. The structures of those samples are considered to be homogeneous in optical level. In DSC measurement only glass transition was observed at relatively low temperature. In DMTA spectra also, unionized samples showed single transition followed by rubbery plateau, suggesting that they have homogeneous amorphous structures. It is considered that cross-linking (in all samples) and side chain (in PN

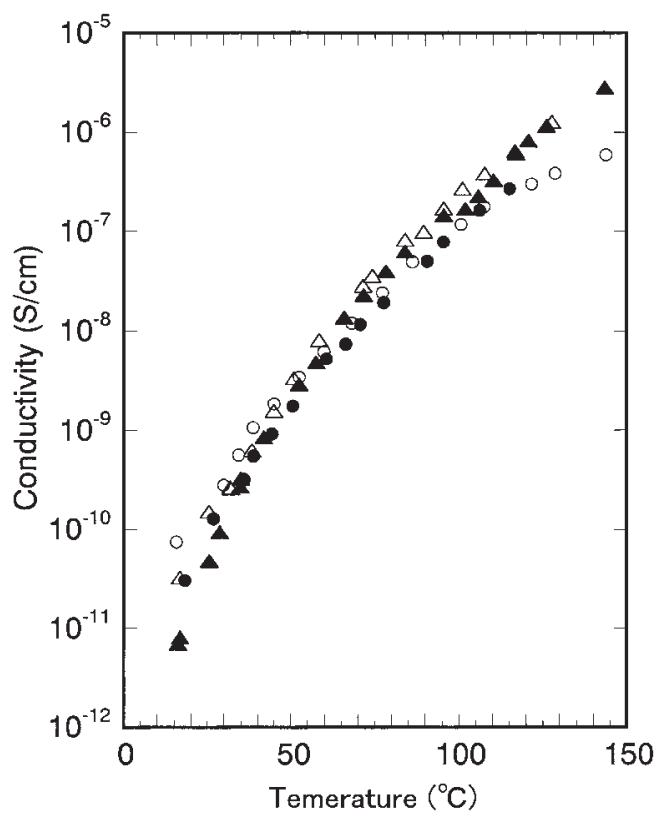

Figure 5. Temperature dependence of ionic conductivity for cationic polyurethane elastomers with 0.6 ionization levels: -10T-PN; O10T-BN; $\Delta 10 \mathrm{P}-\mathrm{PN} ; \triangle 10 \mathrm{P}-\mathrm{BN}$.

samples) prevent aggregation of hard segments.

Reflecting the flexibility of polyether chain, $T_{\mathrm{g}}$ and the corresponding $T_{\alpha}$ of unionized 10T samples (PN and $\mathrm{BN}$ ) is considerably lower than those of unionized $10 \mathrm{P}$ samples ( $\mathrm{PN}$ and $\mathrm{BN}$ ).

By making comparison between PN samples (10T and 10P) and BN samples (10T and 10P), both the $T_{\mathrm{g}}$ and $T_{\alpha}$ stay respectively at almost same temperature range in the case of $10 \mathrm{~T}$ samples. In the case of $10 \mathrm{P}$ samples, PN sample showed slightly higher temperature than that of BN sample. This is probably because the mixing of PU hard segments with polyether soft segments is promoted by the shorter length of chain extender (EMP) than that of BD.

The slopes of the transition region in DMTA spectra of 10P samples are steep, which is typical for amorphous homogeneous structure. Compared with 10P samples, 10T samples showed relatively gentle slope. Because the temperature width of transition region is related to the wideness of the distribution of relaxation mechanisms, the values of temperature range of $T_{\mathrm{g}}, \Delta T_{\mathrm{g}}$, of $10 \mathrm{~T}$ samples are considerably larger than those of 10P samples. As for 10T samples, $\Delta T_{\mathrm{g}}$ of PN sample is larger than that of BN sample, suggesting the increased inhomogeneity. As for 10P samples, there is no difference of $\Delta T_{\mathrm{g}}$ between PN and $\mathrm{BN}$ sample.

From these observations, it is considered that though both the unionized 10T and 10P samples have amorphous homogeneous structure, 10T samples have a little inhomogeneity in soft micro-phase compared with 10P samples, and that PN sample of 10T has en- 
hanced inhomogeneity compared with BN sample of 10T.

\section{Microstructure of Ionized Samples}

In this study ions were introduced by substituting chain extender from EMP to TMPI in PN series and from $\mathrm{BD}$ to MEAI in BN series.

As the content of ion increased, transparency of the samples decreased. It is suggested that the inhomogeneity increased by the aggregation of ionic segments through coulomb force. However, the opacity is different between polyether species. The opacity of $10 \mathrm{~T}$ series is larger than that of 10P series. Compared with PTMG chain, PPG chain with shorter spacing of ether groups is considered to have larger power of mixing with ion-containing segments. Opacity of PN series that contain ion at pendant chain is enhanced compared with $\mathrm{BN}$ series that contain ions at backbone chain. This seems to be the result of enhanced aggregation of hard segment with ion-containing pendant and enhanced micro-phase separation.

Among the DSC results in Table III, $T_{\mathrm{g}}$ is originated from the soft micro-phase rich in polyether segment. Though, in general, the introduction of ion has the effect of elevation of $T_{\mathrm{g}}$ due to the decrease of chain flexibility and to the increase of interchain interaction, $T_{\mathrm{g}}$ lowered with increasing ion content for every series. $T_{\alpha}$ observed in DMTA also lowered in the similar manner.

As for 10T series, with increasing ion content, $T_{\mathrm{g}}$ 's of both the PN and BN series lowered and approached that of pure PTMG $\left(-80^{\circ} \mathrm{C}\right)$. At the low level of ion content, $T_{\mathrm{g}}$ 's are in the same level, but at 0.6 series $T_{\mathrm{g}}$ of $\mathrm{PN}$ is lower than that of $\mathrm{BN}$. As for $10 \mathrm{P}$ series, the lowering of $T_{\mathrm{g}}$ with increasing ion content is small. It is probably because $10 \mathrm{P}$ series have relatively high polarity so that the demixing will not proceed appreciably.

The results mean that in parallel with the diversification of the structures of micro-phases, the purity of polyether in soft micro-phase increases through enhanced demixing of hard segment (ion-containing segment and PU segment). The extent of demixing of hard segment from soft polyether micro-phase depends on sample series.

The hard segment content and the structure of PTMG-rich micro-phase seem to be various. Though $\Delta T_{\mathrm{g}}$ corresponds to the diversity of transition mechanisms in soft segment-rich micro-phase, the situation is observed more clearly in DMTA spectra. The relation of the width of transition region to the increase of ion content differs among sample series. As for 10T series, the transition region has much more gentle slope and much wider temperature range compared with that of unionized sample. As for 10P series, the slope of transition region remains slightly gentler than that of unionized sample.

The situation of $E^{\prime}$ after transition remarkably differs between PN and BN series. As for both the 10T and $10 \mathrm{P}$ series, $E^{\prime}$ of PN series do not show clear rubbery plateau and continues to decrease until flow region. After transition the slope of $E^{\prime}$ of 10T-PN succeeds to that of transition region, and the slope of 10P-PN becomes much gentler than that of transition region. In contrast to PN series, BN series show clear rubbery plateau.

Transition intensity of $T_{\mathrm{g}}$, which is $\triangle C p$, corresponds to the quantity of the phase that undergoes transition, that is, the quantity of transition mechanisms that undergo transition. The relation between the value of $\triangle C p$ and the increase of ion content also depends on sample species. As for 10T series, $\Delta C p$ 's decrease with increasing ion content. As for $10 \mathrm{P}$ series, the value of $\triangle C p$ of PN series does not vary between 0.2 and 0.4 but that of 0.6 decreases. Reversely, the value of BN series slightly increases. That is, as for $10 \mathrm{~T}$ series the quantity of polyether-rich soft micro-phase decreases owing to the demixing of hard segment. As for 10P series, the quantity of PN series tends to decrease and that of $\mathrm{BN}$ series tends to increase.

The temperature of the beginning of flow region of $\mathrm{PN}$ series is lower than that of $\mathrm{BN}$ series.

From these, the following are considered.

(1) As for $10 \mathrm{~T}$ series, as the ion content increases, the demixing of polar (including ionic) hard segment from PTMG soft segment proceeds to various extent. The distribution of inhomogeneity of micro-phases becomes broad. On the one hand aggregation of hard segment proceeds, on the other hand PTMG-rich soft micro-phase becomes purer but its quantity decreases. The extent of the above structural change of PN series is greater than that of BN series.

(2) As for 10P series, as the ion content increases, the similar structural change occurs but its extent is smaller than that of $10 \mathrm{~T}$ series.

(3) PN series of both the 10T and 10P have the micro-multiphase structure in which the extent of micro-aggregation of hard segments continuously varies from low to high, but the strength of aggregation is weak. Contrary to this, BN series have the structure in which the mixing of soft with hard segments proceeds to a considerable extent and give homogeneous rubbery phase after glass transition.

\section{Ionic Conductivity}

In general, ${ }^{20}$ the ionic conductivity $(\sigma)$ in solid is expressed by eq. 2 


$$
\sigma=n q \mu
$$

where $n$ is carrier concentration, $q$ charge, and $\mu$ mobility of carrier ion. In order to increase $\sigma$, it is necessary to increase $n$ and $\mu$. To increase $\mu$, the transportation of ion in polymer should become easy. Generally, the micro-Brownian motion of polymeric chain with low $T_{\mathrm{g}}$ is active and promotes the transportation of ion. To increase $n$, it is necessary to increase the number of introduced ion and to give the structure that promotes the dissociation of ion pair. So far as the structure of ion is the same, the dissociation of ion pair will be enhanced by increasing the dielectric constant of matrix. Therefore, the increase of polar groups such as ether group will increase $n$. Accordingly, if the system is isotropic it is expected that ionic conductivity will be increased by the increase of ion content, the increase of the dielectric constant of matrix, and the lowering of $T_{\mathrm{g}}$.

In this study, the change of conductivity with increasing ion concentration at $25^{\circ} \mathrm{C}$ is considered as follows.

As for 10T-PN series, the conductivity does not change appreciably with the change of ion concentration. $T_{\mathrm{g}}$ is lowered with increasing ion concentration, but the lowering of $T_{\mathrm{g}}$ is the result of inhomogeneity brought by demixing and ion aggregation. The inhomogeneity is promoted with increasing ion concentration and will cause the decrease of conductivity. The actual conductivity will be determined by these contradictory effects.

As for 10T-BN series, the conductivity decreases with increasing ion concentration. Since the inhomogeneity of 10T-BN series is not so large as that of 10T-PN series, 10T-BN series show relatively high conductivity at 0.2 of ion concentration. However, as ion concentration increases, the inhomogeneity of structure is promoted and the conductivity decreased.

As for 10P series, the conductivity of both the PN and $\mathrm{BN}$ series increases with increasing ion concentration. It is considered that the inhomogeneity of the structure is not significant. Though 10P series have larger polarity compared with $10 \mathrm{~T}$ series, which make the dissociation of ion pair easier and inhomogeneity of structure weaken, the conductivity is low. This is considered to be due to the high $T_{\mathrm{g}}$.

As temperature is elevated from 25 to $100{ }^{\circ} \mathrm{C}$, the conductivity of all series increases about 1000 times, reaching equally high level. Two things are noted from the result of $100{ }^{\circ} \mathrm{C}$. Firstly, though the conductivity of $10 \mathrm{~T}-\mathrm{BN}$ series at $25^{\circ} \mathrm{C}$ decreases with increasing ion content, it increases at $100{ }^{\circ} \mathrm{C}$. That is, at $100^{\circ} \mathrm{C}$ the conductivity of all series tends to increase with increasing ion content. As seen in Figure
4, BN samples are in rubbery state and the aggregated structures of PN samples are mostly loosened at this temperature. It is considered that the micro-Brownian motion of polymer chain becomes so active that the effect of $T_{\mathrm{g}}$ can be ignored. The loosening of aggregated structure may also contribute to the increase of conductivity. Owing to these reasons, the conductivity of all the series is considered to be governed basically by the increase of ion content as in homogeneous structure.

Secondly, contrary to the result at $25^{\circ} \mathrm{C}$ where $10 \mathrm{~T}$ series showed higher conductivity than that of $10 \mathrm{P}$ series, the situation becomes reverse at $100{ }^{\circ} \mathrm{C}$ and $10 \mathrm{P}$ series show slightly higher value. This is also explained as the result that the 10T series approximate homogeneous structure as well as 10P series and the larger dielectric constant of PPG matrix forms more effective carrier ion.

We can summarize the situation as follows. In inhomogeneous system, the effective $n$ will not necessarily be proportional to the stoichiometric ion content. On the other hand, because $\mu$ will vary in each microphase of micro-multiphase structure, overall $\mu$ will not be simply determined by observed $T_{\mathrm{g}}$, which is the $T_{\mathrm{g}}$ of soft segment-rich phase.

Though the conductivity of PU-salt complex systems has been discussed simply on the basis of added quantity of salt $(n)$ and $T_{\mathrm{g}},{ }^{21-23}$ it is necessary to elucidate the micro-multiphase structure for the examination of conductivity in the case of ionic PUs.

The temperature dependence of ionic conductivity does not follow the Arrhenius equation. However, the results could be interpreted by using the Vogel ${ }^{24}$ Tamman $^{25}$-Fulcher ${ }^{26}$ (VTF) equation (eq. 3), which has been applied to the inorganic molten salt ionic conductor:

$$
\sigma=\frac{A}{T^{1 / 2}} \exp \left\{\frac{-E_{\mathrm{a}}}{R\left(T-T_{0}\right)}\right\}
$$

where $\sigma$ is ionic conductivity, $T_{0}$ ideal glass transition temperature, $T$ measurement temperature, $R$ gas constant, $A$ a constant, and $E_{\mathrm{a}}$ an apparent activation energy. From the plots of $\log \left(\sigma \cdot T^{1 / 2}\right)$ against $1 /\left(T-T_{0}\right)$, good straight line fit was observed as shown in Figure 6. The good fitting of VTF equation is in general for PU-electrolyte complexs. ${ }^{22,23}$ Ionic PU elastomer in this study showed it similarly. The fact that the temperature dependence of the ionic conductivity obeys the VTF equation suggests that it follows free volume theory, that is, the transportation of carrier ions in ionic network polymer occurs through free volume, which is correlated with the movement of its segments. 


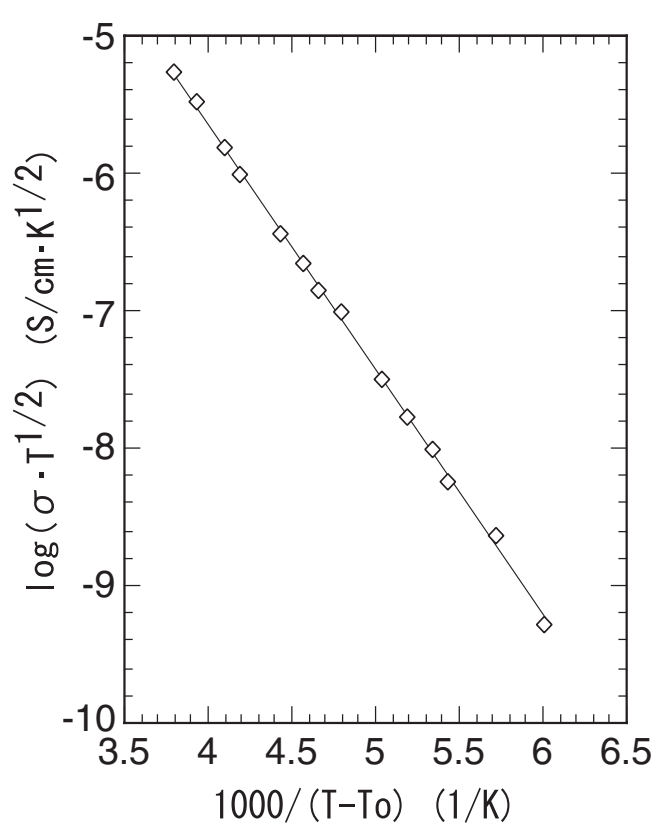

Figure 6. Voyel-Tamman-Fulcher plot for 10T-PN-0.6.

\section{CONCLUSION}

A series of cationic polyurethane elastomers with different level of ionization were prepared by using cationic short-chain diol with pendant trimethylammonium group, and their thermal and mechanical properties and ionic conductivity were investigated in comparison with cationic PU elastomer having quaternary ammonium group in backbone. From these measurements, the following results were obtained;

(1) Thermosetting cationic PU elastomers with pendant trimethylammonium group were successfully synthesized by nonsolvent technique by using prequarternized chain extender.

(2) The difference of polyol types has greater effect on properties of cationic PU elastomers compared with ionic sites.

(3) The heterogeneity of cationic PU elastomers with pendant trimethylammonium group is larger than that with ammonium group in backbone.

(4) The ionic conductivity of cationic PU elastomers with micro-phase separation, which is not simple as that with homogeneous structure, is interpreted by considering the effects of structure on the concentration and the mobility of carrier ion.

(5) Temperature dependence of the conductivity of cationic PU elastomers obeys VTF equation, suggesting that the transportation of carrier ions occurs through free volume.

\section{REFERENCES}

1. M. Seki, K. Sato, and R. Yosomiya, Makroml. Chem., 193, 2971 (1992).

2. M. Watanabe, M. Itoh, K. Sanui, and N. Ogata, Macromolecules, 20, 569 (1987).

3. D. Dieterich, W. Keberle, and H. Witt, Angew. Chem. Int. Ed., 9, 40 (1970).

4. S. Kohjiya, T. Ohtsuki, and S. Yamashita, Makromol. Chem., Rapid. Commun., 2, 417 (1981).

5. H. A. Al-Salah, H. X. Xiao, J. A. McLean, Jr., and K. C. Frisch, J. Polym. Sci., Polym. Chem. Ed., 26, 1609 (1988).

6. S.-A. Chen and W.-U. Chan, J. Polym. Sci., Polym. Phys. Ed., 28, 1499 (1990).

7. S.-A. Chen and W.-U. Chan, J. Polym. Sci., Polym. Phys. Ed., 28, 1515 (1990).

8. D.-C. Lee, R. A Register, C.-Z. Yang, and S. L. Cooper, Macromolecules, 21, 998 (1988)

9. H.-S. Xu and C.-Z. Yang, J. Polym. Sci., Polym. Phys. Ed., 33, 745 (1995).

10. X.-H. Yu, A. Z. Okema, and S. L. Cooper, J. Appl. Polym. Sci., 41, 1777 (1990).

11. J. D. Van Heumen and J. R. Steens, Macromolecules, 28, 4268 (1995).

12. C. Wang and S. Nakamura, J. Polym. Sci., Polym. Chem. Ed., 32, 1255 (1994).

13. V. A. Vilenskii, Y. Y. Kercha, Y. S. Lipatov, V. I. Shtompel, V. A. Shrubovich, and V. V. Shevchenko, Docklady Phys. Chem., 281, 370 (1985).

14. S. C. Varma, M.A. Ahsan, M.H. George, and J. A. Barrie, Polym. Commun., 31, 11 (1990).

15. R. J. Goddard and S. L. Cooper, J. Polym. Sci., Polym. Phys. Ed., 32, 1557 (1994).

16. R. J. Goddard and S. L. Cooper, Macromolecules, 28, 1390 (1995).

17. R. J. Goddard and S. L. Cooper, Macromolecules, 28, 1401 (1995).

18. M. Takesue, K. Hiraoka, and T. Yokoyama, Nippon Gomu Kyokaishi, 69, 491 (1996).

19. M. Takesue, K. Hiraoka, K. Ashizuka, and T. Yokoyama, Kobunshi Ronbunshu, 55, 802 (1998).

20. N. Ogata, Ed., "Doudensei Kobunshi”, Kodansha Co., Ltd., Tokyo, 1990, p.129.

21. M. Watanabe, M. Itoh, K. Sanui, and N. Ogata, Macromolecules, 20, 569 (1987).

22. H. Tada, K. Fujino, and H. Kawahara, J. Polym. Sci., Polym. Chem. Ed., 25, 3015 (1987).

23. A. Ferry, P. J. Jacobsson, J. D. van Heumen, and J. R. Stevens, Polymer, 37, 737 (1996).

24. H. Vogel, Phys. Z., 22, 645 (1921).

25. G. Tamman and W. Hesse, Z. Anorg. Allg. Chem., 156, 245 (1926).

26. G. S. Fulcher, J. Am. Ceram. Soc., 8, 339 (1925). 